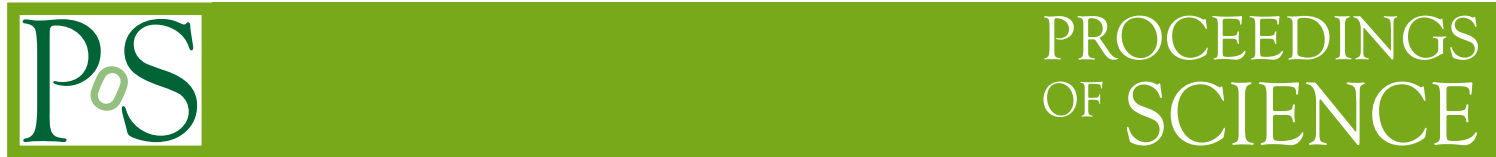

\title{
The Crab Nebula in the infrared: a review
}

\author{
Paolo Persi \\ Author affiliation \\ INAF/IASF Rome - Italy \\ E-mail: paolo.persi@iasf-roma.inaf.it
}

A review of the Crab Nebula in the infrared including ground based near-infrared and space observations obtained with the ISO and Spitzer satellites is presented. A brief discussion about the infrared polarimetry of the Crab is also reported.

Polarimetry days in Rome: Crab status, theory and prospects October 16-17 2008

Rome, Italy 


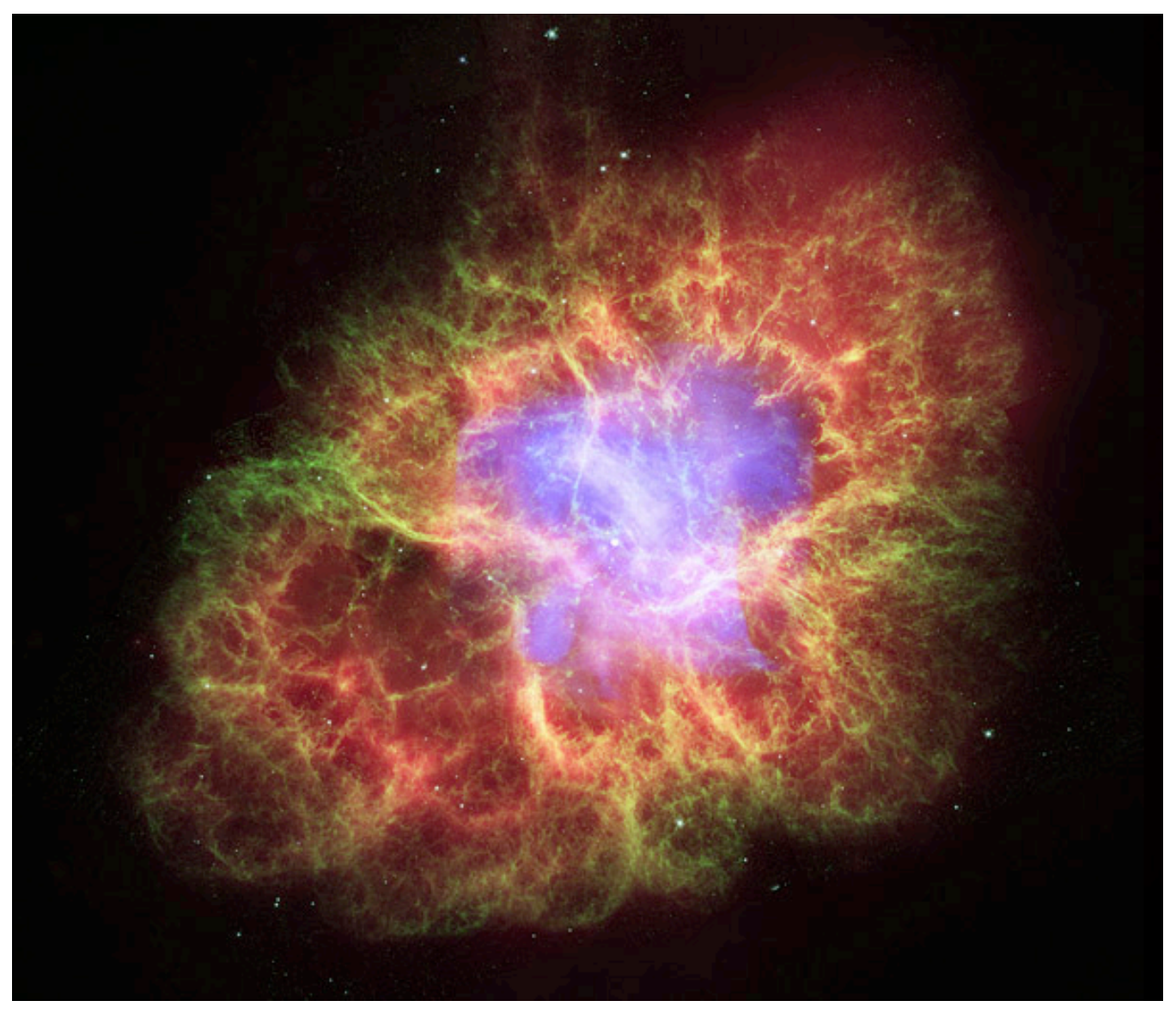

Figure 1: Composite image of th Crab Nebula obtained with the Chandra X-ray image (blue), Hubble telescope optical image (green), and the Spitzer infrared image (red) .

\section{Introduction}

The Crab Nebula is one of the brightest synchrotron source in the Galaxy. It is a prototype of a class of SNRs called plerions, which are powered by a central pulsar. The supernova which produced the Crab Nebula is located in the costellation of Taurus at a distance of about $2 \mathrm{kpc}$.

Comparing the X-ray, optical and infrared images obtained respectively with Chandra, Hubble Space Telescope and the Spitzer Space telescope (see the composite image of Figure 1), results that the nebula appears most compact in X-rays and largest in the infrared. This can be understood by the fact that ultrahigh-energy X-ray emitting electrons radiate away their energy more quickly than the lower-energy electrons emitting optical and infrared radiation.

One of the main reasons to observe the Crab Nebula in the infrared is to test the hypothesis that part of the interstellar dust does originate from SNe. In fact the infrared (IR) is an ideal spectral region in which to test these theories because dust grains emit strongly in the IR. In addition the comparison of the spectral index derived from IR observations with those obtained in the radio and UV-Opt. spectral regions, is foundamental in understanding the physical mechanisms of emission in the Crab Nebula.

In Sect. 2 will be presented a review of the main infrared observations of the Crab Nebula, 

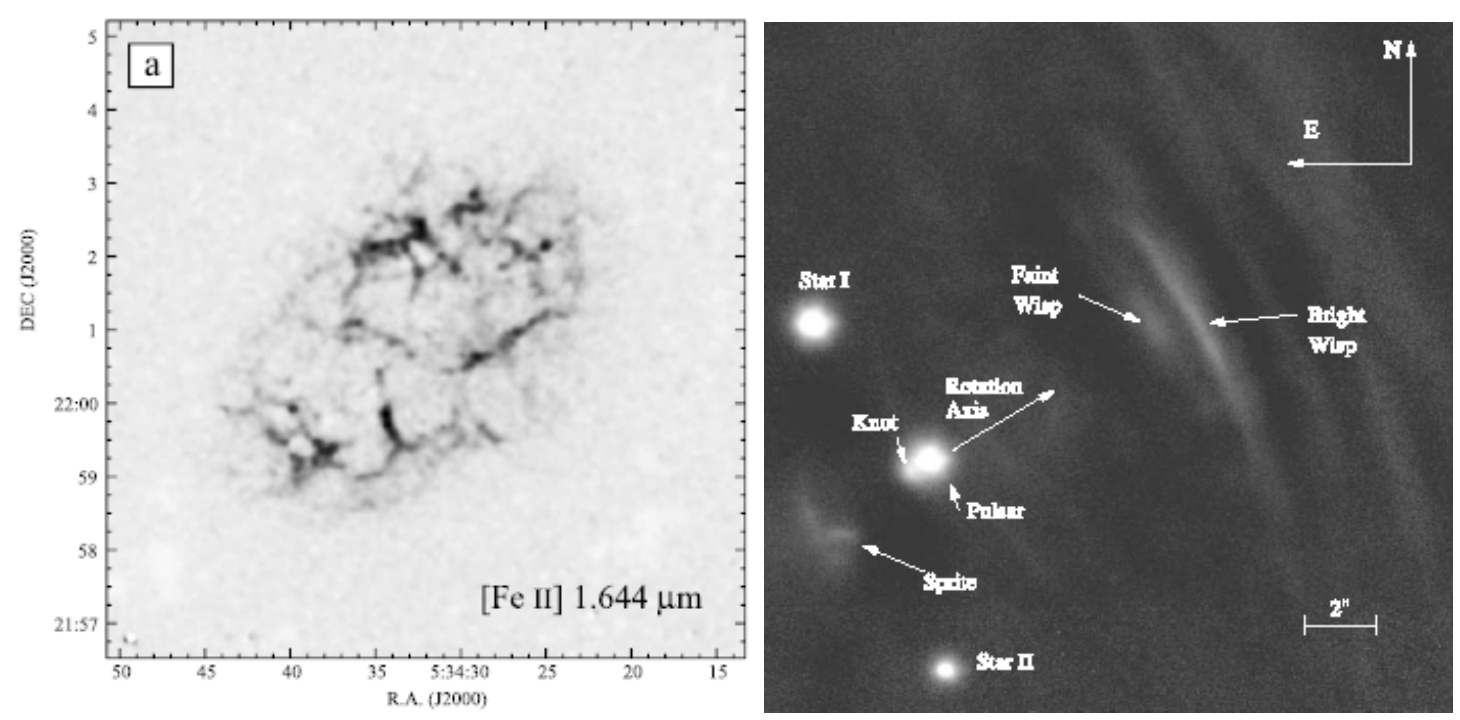

Figure 2: Left panel: [Fe II] $1.644 \mu \mathrm{m}$ image of the Crab Nebula [4]. Right panel: Sub-arcsec K' image of the central $20 \operatorname{arcsec} \times 20$ arcsec region of the Crab pulsar [5]. The principal features of the pulsar wind termination shock and its environment are labeled.

while in Sect. 3 are reported the recent Spitzer images. Polarimetric infrared observations are briefly described in Sect.4, and in Sect.5 a summary is reported.

\section{Infrared observations}

\subsection{Near-Infrared}

Near-infrared photometric observations of the Crab Nebula were obtained in the past by different authors (see i.e. [1], [2]). From the dereddened spectral energy distribution obtained combining the observed integrated flux densities from 1-5 $\mu \mathrm{m}$ with optical data, a spectral index of $\alpha=-0.46$ $\left(\mathrm{f}_{v} \propto v^{\alpha}\right)$ was derived ([2]). This value supports the synchrotron mechanism in the near-IR. In addition, the first image at $2.2 \mu \mathrm{m}$ obtained by [2] showed a strict correlation with the visible flux distribution.

With the advent of the near-IR array detectors has been possible to study in details the morphology of the nebula and the central pulsar. Particularly interesting are the $1.644 \mu \mathrm{m}$ images centered on the [Fe II] line obtained by [3] and [4] (left panel of Fig.2). These images are dominated by a prominent filament structure identical to the [Ni II] image indicating that the gas phase distribution of $\mathrm{Fe}^{+}$and $\mathrm{Ni}^{+}$are very similar. In two of the filaments, [6] report the discovery of $\mathrm{H}_{2}$ emission at $2.122 \mu \mathrm{m}$. This discovery raises fashinating questions about the origin, survival, and excitation of the molecular gas in the Crab Nebula. New high resolution images centered on the $\mathrm{H}_{2}$ line and nearby continuum, have been recently obtained at the Telescopio Nazionale Galileo (TNG) using the NICS camera in order to detect hydrogen emission knots in the central part of the Crab. The results of these observations will be presented in a forthcoming paper.

Sub-arcsec resolution image in the continuum at $2.2 \mu \mathrm{m}$ ([5]) shows the presence of bright and faint equatorial wisps and a polar knot (right panel of Fig.2) around the central pulsar. These 

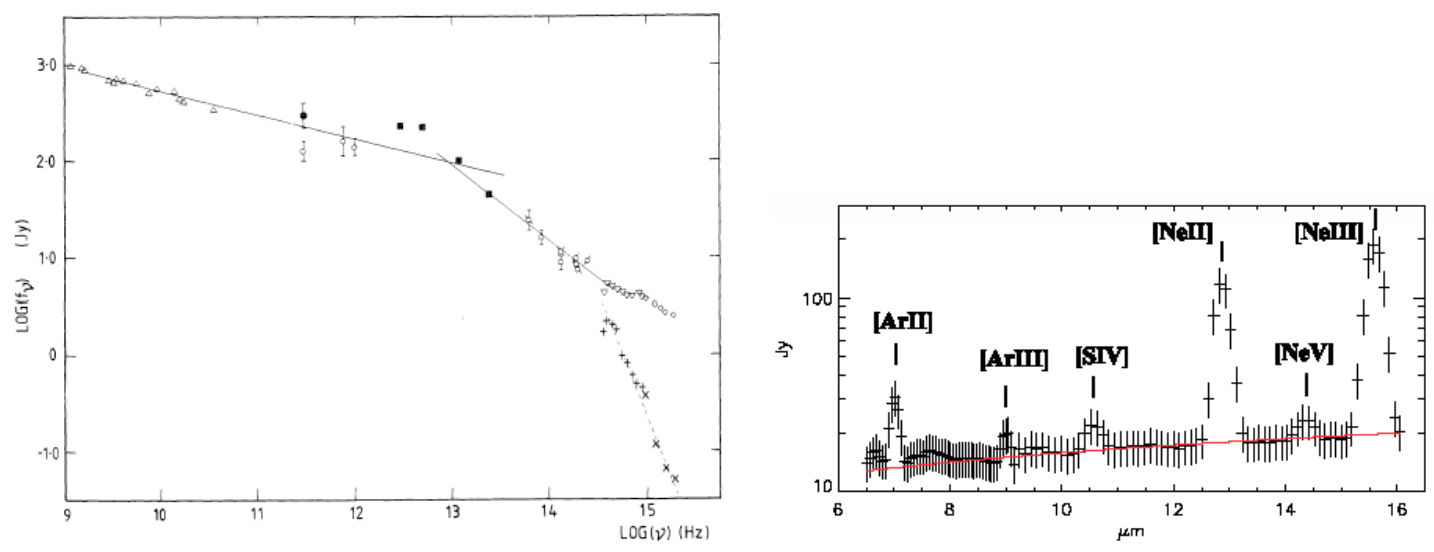

Figure 3: Left panel: Spectral energy distribution of the Crab Nebula from UV to radio spectral region [7]. Right panel: Mid-IR spectrum of the Crab Nebula. The spectrum features ionic forbidden lines superimposed onto a flat continuum synchrotron emission. The full line represents the spectrum of the synchrotron radiation with a spectral index of -0.5 ([10]).

features appear to fluctuate in brightness on kilosecond timescales.

\subsection{Mid and Far-Infrared}

The Crab Nebula was observed between 12 and $100 \mu \mathrm{m}$ with IRAS ([7]). The observations show an infrared excess beyond $12 \mu \mathrm{m}$ over the extraplolated radio and near-IR fluxes peaking between 60 and $100 \mu \mathrm{m}$ (left panel of Fig.3). This has been interpreted as due to thermal radiation by dust at temperature of $46 \mathrm{~K}$ and with mass of $0.02 \mathrm{M}_{\text {sun }}$ ([8]). Images at 60 and $100 \mu \mathrm{m}$ taken

with ISOPHOT at higher spatial resolution reveal that about half this excess is attributable to two peaks, separated by $\sim 80$ arcsec [9].

Spectro-imaging observations between 6.5 and $16.5 \mu \mathrm{m}$ of the central $3 \operatorname{arcmin} \times 3 \operatorname{arcmin}$ part of the nebula at a spatial resolution of 6 arcsec were obtaind with ISOCAM by [10]. Ionic lines of [Ne II], [Ne III], [Ne V] at respectively 12.8, 15.5 and $14.3 \mu \mathrm{m}$, of [Ar II], [Ar III] at 7.0 and $9.0 \mu \mathrm{m}$ and of [S IV] at $10.5 \mu \mathrm{m}$ were detected (see right panel of Fig.3).

The image centered on the [NeIII] emission shows a filamentary structure, similar to the structure seen in the optical ionic lines. These filaments are known to be composed predominately of supernova ejecta. Completely different is the morphology of the nebula in the continuun at about $11.3 \mu \mathrm{m}$ that is similar to the synchrotron nebula seen at other wavelengths (radio, optical, Xrays). Threfore, the mid-IR emission from the Crab is dominated by synchrotron radiation; no dust is detected in this spectral region.

\section{SPTZER Images}

Images of the Crab Nebula at 3.6, 4.5, 5.8, 8.0, 24, and $70 \mu \mathrm{m}$ were obtained with the Spitzer Space Telescope IRAC and MIPS cameras ([4]). Figure 4 shows the four IRAC images (panel $\mathrm{c}, \mathrm{d}, \mathrm{e}$, and f) of the central part of the nebula. The pulsar, and the associated equatorial toroid and polar jet structures seen in Chandra and Hubble Space Telescope images (panel a, and b in Fig.4) can be identified in all of the IRAC images. The $8.0 \mu \mathrm{m}$ image, including the [Ar II] $7.0 \mu \mathrm{m}$ line, 

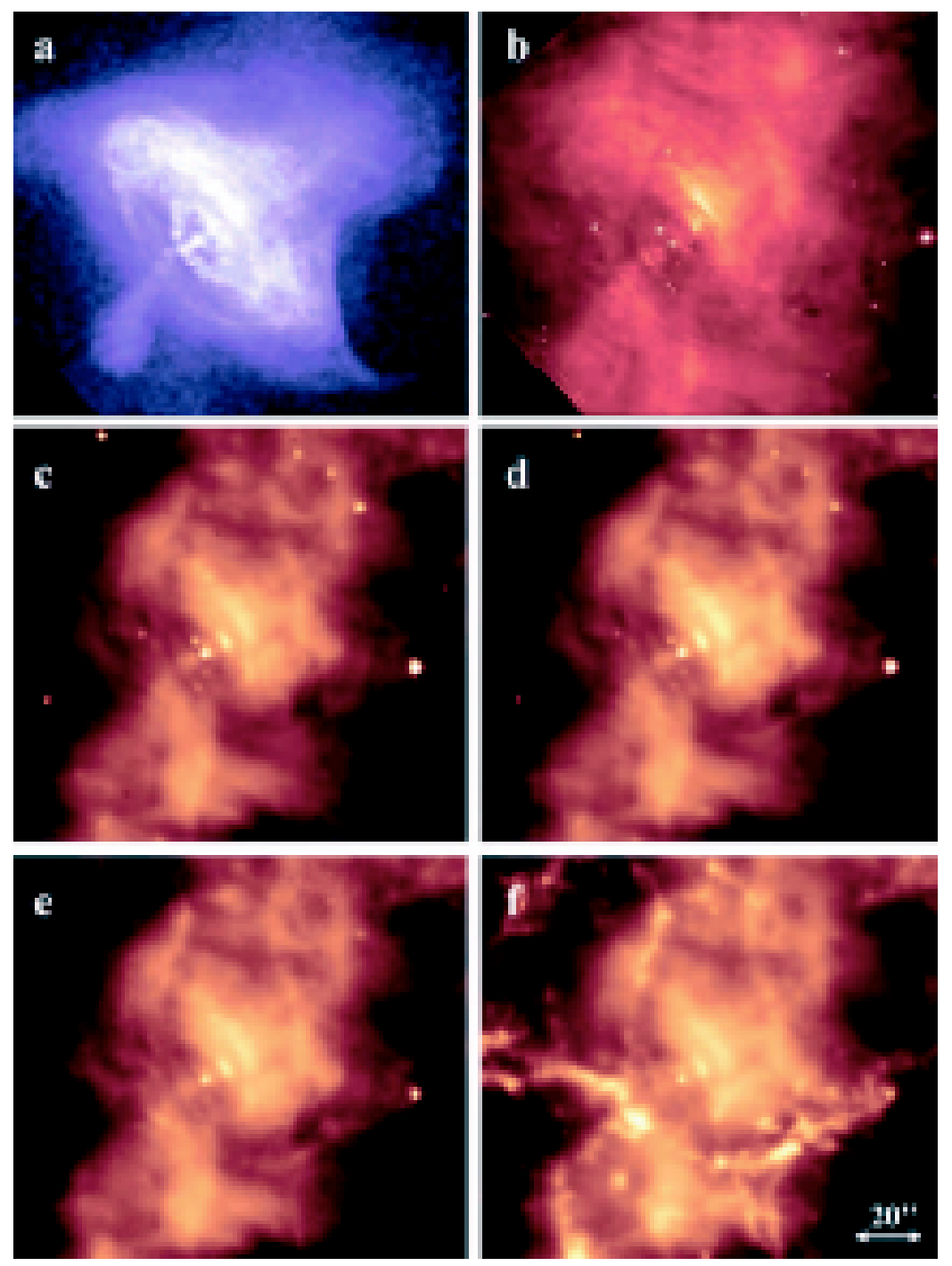

Figure 4: SPITZER/IRAC images at 3.6, 4.5, 5.8 and $8.0 \mu \mathrm{m}$ (panels c,d,e, and f respectively) of the central part of the Crab Nebula. The Chandra X-ray ACIS-S and HST optical images are reproduced for comparison in panels a and $b([4])$

resembles the general morphology of visible $\mathrm{H}_{\alpha}$ and near-IR [Fe II] line emission, while the 3.6 and $4.5 \mu \mathrm{m}$ images are dominated by continuum synchrotron emission.

The 24 and $70 \mu \mathrm{m}$ images show enhanced emission that may be due to line emission or the presence of a small amount of warm dust in the nebula on the order of less than $1 \%$ of a solar mass.

\section{Infrared polarimetry}

Polarimetric imaging observations in differen spectral regions, remain central to an understanding of the structure and evolution of the Crab Nebula. The only infrared polarimetric observations of the Crab Nebula have been obtained with the ISOCAM at 6.7 and $14.3 \mu \mathrm{m}$ ([11]) and ISOPHOT at 25 and $170 \mu \mathrm{m}$ ([12]). The preliminary results reported by [11] indicate that the pat- 

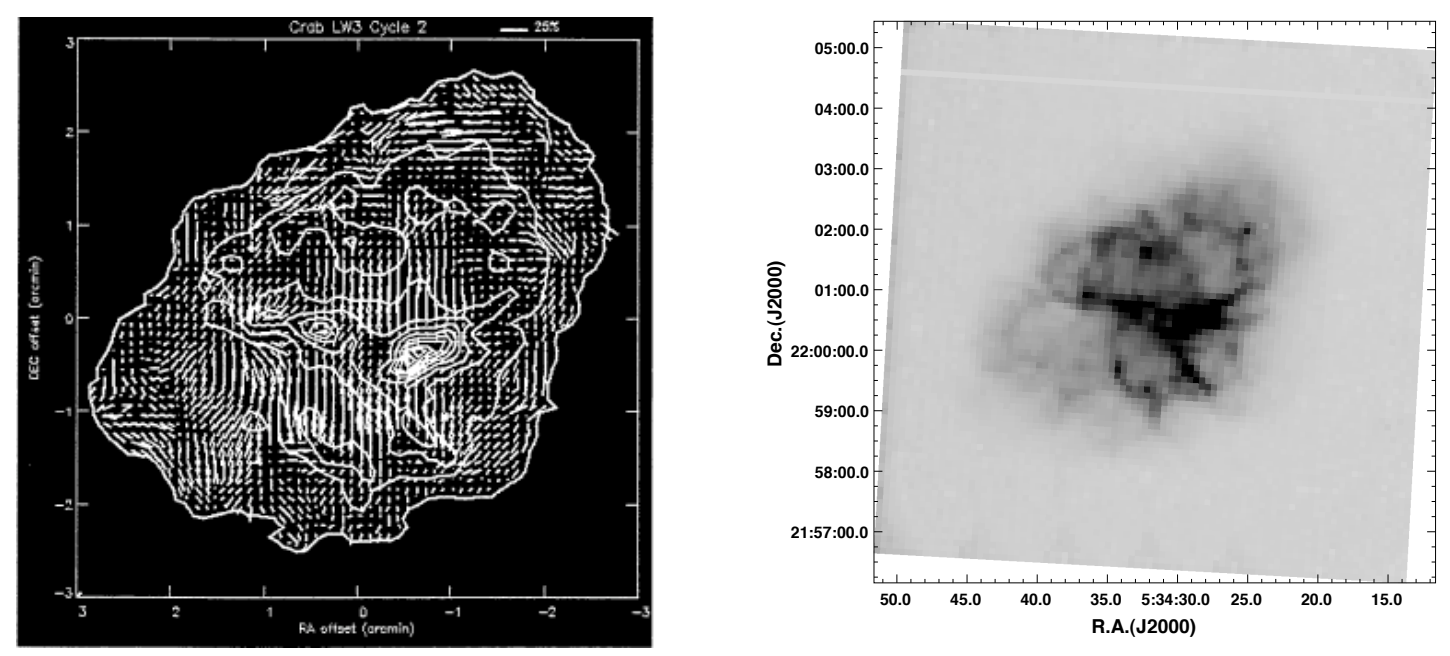

Figure 5: Left panel: Electric vectors of polarised intensity overlayd on contours of the ISOCAM image at $14.3 \mu \mathrm{m}$. ([11]. Right panel: ISOCAM image at $14.3 \mu \mathrm{m}$.

tern of spatially coherent vectors shows some similaty to that seen in the radio and optical regimes (left panel of Fig.5). The polarimetry at $170 \mu \mathrm{m}$ obtained by [12] gives a preliminary polarization intensity $p=8.2 \%$ with an orientation angle $\Theta=103^{\circ}$ at the position of the peak polarized intensity.

Further polarimetric observations in the near-IR are planned at the italian TNG telescope in order to compare the polarimetry of the Crab Nebula in different spectral regions.

\section{Summary}

In this paper the main infrared observations of the Crab Nebula are discussed. From these observations, the following conclusions can be derived:

a) The near and mid-IR continuum in the Crab Nebula is dominated by synchrotron emission. Ionic lines have been observed in these spectral regions with a filamentary structure similar to that observed in the optical lines.

b) A break in the spectral energy distribution between 60 and $100 \mu \mathrm{m}$ suggests the presence of dust at a temperature of $46 \mathrm{~K}$.

c) The structures observed in the optical and X-ray associated with the pulsar are also present in the near-IR untill $8 \mu \mathrm{m}$.

d) More polarimetric observations, specially in the near-IR are request in order to understand the mechanism of emission in the central part of the nebula.

\section{References}

[1]Becklin, E.E., \& Kleinmann, D.E. 1968, ApJ, 152, L52

[2]Grasdalen, G.L. 1979, PASP, 91, 436

[3]Herster, J.J., Graham, J.R, Beichman, C.A. \& Gautier, T.N. 1990, ApJ, 357, 539 
[4]Temim, T., Gehrz, D., Woodward, C.E. \& et al. 2006, AJ, 132, 1610

[5]Melatos, A., Scheltus, D., Whiting, M.T., \& et al. 2005, ApJ, 633, 931

[6]Graham, J.R., Wright, G.S., \& Longmore, A..J. 1990, ApJ, 352, 172

[7]Marsden, P.L., Gillett, F.C., Jennings, J.P, \& et al. 1984, ApJ, 278, L29

[8]Strom, R.G., \& Greidanus , H. 1992, Nature, 358, 654

[9]Green, D.A., Tuff, R.J., \& Popescu , C..C. 2004, MNRAS, 355, 1315

[10]Douvion, T., Lagage, P.O., Cesarsky, C.J. \& Dwek, E. 2001, A\&A, 373, 281[11]

[11]Tuffs, R.J., Siebenmorgen, R., \& Gallant, Y..A. 1999, ESA SP-435, p.47

[12]Klaas, U., Muller, T.G., Laureijs, R.J.,\& Clevel , J. 1999, ESA SP-427, p.77 